\title{
$\mu$-statistical convergence and the space of functions $\mu$-stat continuous on the segment
}

\begin{abstract}
Sadigova S.R.
In this work, the concept of a point $\mu$-statistical density is defined. Basing on this notion, the concept of $\mu$-statistical limit, generated by some Borel measure $\mu(\cdot)$, is defined at a point. We also introduce the concept of $\mu$-statistical fundamentality at a point, and prove its equivalence to the concept of $\mu$-stat convergence. The classification of discontinuity points is transferred to this case. The appropriate space of $\mu$-stat continuous functions on the segment with sup-norm is defined. It is proved that this space is a Banach space and the relationship between this space and the spaces of continuous and Lebesgue summable functions is considered.

Key words and phrases: $\mu$-stat convergence, $\mu$-stat fundamentality, space of $\mu$-statistical continuous functions.
\end{abstract}

Institute of Mathematics and Mechanics of NAS of Azerbaijan, Baku, Azerbaijan; Khazar University, Baku, Azerbaijan E-mail: s_sadigova@mail.ru

\section{Introduction}

Actually, the concept of statistical convergence of the sequences of complex numbers has long been known as "almost convergence" (see, e.g., the monograph of A. Zygmund [44]). It was introduced in the study of pointwise convergence of the Fourier series of summable functions. Equivalent definition for this concept was given by H. Fast in [14] (see also H. Steinhaus [43]), where it was (for the first time) referred to as "statistical convergence". In [16, 17,38,42], the basic properties of statistically convergent sequences are investigated and are mainly generalized in two directions. The first direction included the generalizations of the concept of statistical convergence itself, so there arose $I$-convergence (ideal convergence), $\mathscr{F}$-convergence (filter convergence), lacunar convergence, etc. (see, e.g., [9-11, 18-21,30,33, 37, 40]).

The second direction treated these kinds of convergence in various mathematical structures (see $[1-6,12,13,22-24,27,28,31,39,41])$. In [26,34,36], the statistical convergence was generalized for double sequences, and the properties of this convergence were studied. The number of all relevant works is too big, and it should be noted that it is impossible to name all of them here.

Quite naturally, there arises the question about the existence of a continuous analog of the concept of statistical convergence for number sequences (or for elements of other mathematical structures). The first step in this direction was made by F. Moricz [32], who introduced the concepts of statistical limit and statistical fundamentality for measurable functions at infinity and at a finite point, generated by the Lebesgue measure. F. Moricz proved the equivalence of these concepts and studied some of their properties. He also studied the relationship between this kind of convergence and the one of Fourier series. But, this concept is not a generalization 
of the similar concept for sequences, because it does not imply, as a special case, the concept of statistical convergence for sequences.

The direct generalization of the concept of statistical convergence in continuous case was first carried out by B.T. Bilalov and S.R. Sadigova [7]. They introduced the concepts of $\mu$-stat convergence and $\mu$-stat fundamentality, proved their equivalence and studied some of their properties. They also introduced the concept of $\mu$-stat continuity. $\mu$-stat convergence is a direct generalization of the statistical convergence in continuous case, as it turns out from this concept as a special case.

It should be noted that the concept of a density point and approximately continuity at a point are known with respect to the Lebesgue measure. In the main, some properties of Lebesgue measurable functions in connection with these concepts are studied, Luzin type, Denjoy type theorems, theorems on belonging to Baire class etc. are proved. More details on this information can be considered in monographs [8, 15,25]. In [29], these concepts are considered with respect to an arbitrary measure. It is proved that any approximately continuous function has the property of Baire. The connection between such functions and measurable functions is found.

We introduce the concepts of $\mu$-stat limit, $\mu$-stat fundamentality and $\mu$-stat continuity, which are the direct generalizations to the continuous case (or to the case of measurable spaces with measure) of the corresponding concepts of the statistical limit and the statistical fundamentality of the sequences of elements. Therefore, we retained these names, in contrast to the name of approximately continuous, and we study the problems dictated by the discrete case.

In the present paper, the concept of a point $\mu$-statistical density is defined. Basing on this notion, the concept of $\mu$-statistical limit, generated by some Borel measure $\mu(\cdot)$, is defined at a point, in contrast to similar concepts [7]. We also introduce the concept of $\mu$-statistical fundamentality at a point, and prove its equivalence to the concept of $\mu$-stat convergence. The classification of discontinuity points is transferred to this case. The appropriate space of $\mu$-stat continuous functions on the segment with sup-norm is defined. It is proved that this space is a Banach space and the relationship between this space and the spaces of continuous and Lebesgue summable functions is considered.

\section{$1 \quad \mu$-stat limit}

We will use the standard notations: $\mathbb{N}$ will be the set of all positive integers; $\mathbb{R}$ is the set of all real numbers; $\exists$ means "there exist(s)"; $\exists$ ! means "there exists a unique"; $\Rightarrow$ will denote "it follows"; $\Leftrightarrow$ will stand for equivalence.

Let $J \subset \mathbb{R}$ be some segment, $\mathscr{B}$ be $\sigma$-algebra of all Borel subsets and $\mu: \mathscr{B} \rightarrow \mathbb{R}_{+}:=[0,+\infty)$ be a Borel measure. Let $M \in \mathscr{B}$ be some set. Put $O_{\delta}(x)=J \cap(x-\delta, x+\delta)$.

Throughout this paper we assume that the measure $\mu(\cdot)$ satisfies the following condition:

$$
\text { ג) } \mu\left(O_{\delta}(x)\right)>0 \& \mu(\{x\})=0, \quad \forall x \in J, \quad \forall \delta>0 .
$$

We say that the point $x_{0} \in J$ is a point $\mu$-stat density for $M$, if

$$
\lim _{\delta \rightarrow 0} \frac{\left|M \cap O_{\delta}^{0}\left(x_{0}\right)\right|}{\left|O_{\delta}^{0}\left(x_{0}\right)\right|}=1,
$$

where $|A|=\mu(A)$ and $O_{\delta}^{0}\left(x_{0}\right)=O_{\delta}\left(x_{0}\right) \backslash\left\{x_{0}\right\}$. 
Let $f: J \rightarrow \mathbb{R}$ be some $(J ; \mathscr{B})$-measurable function and $\varepsilon>0$ be some number. For a given number $l \in \mathbb{R}$ assume

$$
\Delta_{\varepsilon}(f ; l)=\{x \in J:|f(x)-l|<\varepsilon\} .
$$

Denote by $J_{s t}\left(x_{0}\right)$ the family of all sets of $\mathscr{B}$, which $x_{0}$ is the point of $\mu$-stat density.

Definition 1. We say that $l$ is $\mu$-stat limit of the function $f$ at a point $x_{0}$, if $\Delta_{\varepsilon}(f ; l) \in J_{s t}\left(x_{0}\right)$, $\forall \varepsilon>0$, i.e.

$$
\lim _{\delta \rightarrow 0} \frac{\left|\Delta_{\varepsilon}(f ; l) \cap O_{\delta}^{0}\left(x_{0}\right)\right|}{\left|O_{\delta}^{0}\left(x_{0}\right)\right|}=1 .
$$

This limit will be denoted as $\mu$-st $\lim _{x \rightarrow x_{0}} f(x)=l$.

For $M \in \mathscr{B}$ assume $M^{c}=J \backslash M$. Thus, it is clear that

$$
O_{\delta}^{0}\left(x_{0}\right)=\left(\Delta_{\varepsilon}(f ; l) \cap O_{\delta}^{0}\left(x_{0}\right)\right) \cup\left(\Delta_{\varepsilon}^{c}(f ; l) \cap O_{\delta}^{0}\left(x_{0}\right)\right),
$$

where $\Delta_{\varepsilon}^{c}(f ; l)=J \backslash \Delta_{\varepsilon}(f ; l)=\{x \in J:|f(x)-l| \geq \varepsilon\}$. Consequently

$$
\frac{\left|\Delta_{\varepsilon}(f ; l) \cap O_{\delta}^{0}\left(x_{0}\right)\right|+\left|\Delta_{\varepsilon}^{c}(f ; l) \cap O_{\delta}^{0}\left(x_{0}\right)\right|}{\left|O_{\delta}^{0}\left(x_{0}\right)\right|}=1 .
$$

This immediately implies that the relation (1) is equivalent to

$$
\lim _{\delta \rightarrow 0} \frac{\left|\Delta_{\varepsilon}^{c}(f ; l) \cap O_{\delta}^{0}\left(x_{0}\right)\right|}{\left|O_{\delta}^{0}\left(x_{0}\right)\right|}=0 .
$$

Let us show that $\mu$-stat limit $l$ is unique. Assume the opposite: there are two $\mu$-stat limits $l_{1}$ and $l_{2}$. Take $\varepsilon$ such that $0<\varepsilon<\frac{1}{2}\left|l_{1}-l_{2}\right|$. We have

$$
\left[\left(\Delta_{\varepsilon}\left(f ; l_{1}\right) \cap O_{\delta}^{0}\left(x_{0}\right)\right) \cup\left(\Delta_{\varepsilon}\left(f ; l_{2}\right) \cap O_{\delta}^{0}\left(x_{0}\right)\right)\right] \subset O_{\delta}^{0}\left(x_{0}\right) .
$$

Consequently

$$
\left|\Delta_{\varepsilon}\left(f ; l_{1}\right) \cap O_{\delta}^{0}\left(x_{0}\right)\right|+\left|\Delta_{\varepsilon}\left(f ; l_{2}\right) \cap O_{\delta}^{0}\left(x_{0}\right)\right| \leq\left|O_{\delta}^{0}\left(x_{0}\right)\right| .
$$

Hence we arrive at a contradiction

$$
2=\lim _{\delta \rightarrow 0} \frac{\left|\Delta_{\varepsilon}\left(f ; l_{1}\right) \cap O_{\delta}^{0}\left(x_{0}\right)\right|}{\left|O_{\delta}^{0}\left(x_{0}\right)\right|}+\lim _{\delta \rightarrow 0} \frac{\left|\Delta_{\varepsilon}\left(f ; l_{2}\right) \cap O_{\delta}^{0}\left(x_{0}\right)\right|}{\left|O_{\delta}^{0}\left(x_{0}\right)\right|} \leq 1 .
$$

It is absolutely obvious that, if $\lim _{x \rightarrow x_{0}} f(x)=l$, then $\exists \mu$-st $\lim _{x \rightarrow x_{0}} f(x)$ and $\mu$-st $\lim _{x \rightarrow x_{0}} f(x)=l$. The converse is not always true. For example, let $\mu$ be a Lebesgue measure and consider the Dirichlet function on $J$

$$
D(x)= \begin{cases}0, & x \in J \backslash \mathbb{Q}_{J}, \\ 1, & x \in \mathbb{Q}_{J},\end{cases}
$$

where $\mathbb{Q}_{J}$ are rational numbers from $J$. It is absolutely obvious that for all $x_{0} \in J \backslash \mathbb{Q}_{J}$ we have $\mu$-st $\lim _{x \rightarrow x_{0}} f(x)=0$, but for $x_{0} \in \mathbb{Q}_{J}$ a $\mu$-stat limit does not exist.

Since

$$
\lambda \neq 0:\{x:|\lambda f(x)-\lambda l| \geq \varepsilon\} \Leftrightarrow\left\{x:|f(x)-l| \geq \frac{\varepsilon}{|\lambda|}\right\}
$$


then it is clear that

$$
\mu \text {-st } \lim _{x \rightarrow x_{0}}(\lambda f(x))=\lambda\left(\mu \text {-st } \lim _{x \rightarrow x_{0}} f(x)\right) \text {. }
$$

Let $\mu$-st $\lim _{x \rightarrow x_{0}} f_{k}(x)=l_{k}, k=1$, 2 . It is absolutely obvious that

$$
\left\{x:\left|f_{1}(x)+f_{2}(x)-\left(l_{1}+l_{2}\right)\right| \geq \varepsilon\right\} \subset\left[\left\{x:\left|f_{1}(x)-l_{1}\right| \geq \frac{\varepsilon}{2}\right\} \cup\left\{x:\left|f_{2}(x)-l_{2}\right| \geq \frac{\varepsilon}{2}\right\}\right] .
$$

Consequently

$$
\begin{aligned}
\mid\left\{x:\left|f_{1}+f_{2}-\left(l_{1}+l_{2}\right)\right| \geq \varepsilon\right\} & \cap O_{\delta}^{0}\left(x_{0}\right) \mid \\
& \leq\left|\left\{x:\left|f_{1}-l_{1}\right| \geq \frac{\varepsilon}{2}\right\} \cap O_{\delta}^{0}\left(x_{0}\right)\right|+\left|\left\{x:\left|f_{2}-l_{2}\right| \geq \frac{\varepsilon}{2}\right\} \cap O_{\delta}^{0}\left(x_{0}\right)\right| .
\end{aligned}
$$

Hence it directly follows that $\mu$-st $\lim _{x \rightarrow x_{0}}\left(f_{1}(x)+f_{2}(x)\right)=l_{1}+l_{2}$.

Thus, $(J ; \mathscr{B})$-measurable functions with $\mu$-stat limit at the point $x_{0} \in J$ form a linear space over a field $K$, and we denote this space by $\mathscr{B}_{s t}\left(x_{0}\right)$.

Similarly we define the concepts of one-sided $\mu$-stat limits at a point $x_{0}$. Denote

$$
O_{\delta}^{+}\left(x_{0}\right)=\left(x_{0}, x_{0}+\delta\right) \cap J .
$$

Definition 2. We say that $\ell$ is a right-hand $\mu$-stat limit of a function $f$ at a point $x_{0}$ if

$$
\lim _{\delta \rightarrow 0} \frac{\left|\Delta_{\varepsilon}(f ; l) \cap O_{\delta}^{+}\left(x_{0}\right)\right|}{\left|O_{\delta}^{+}\left(x_{0}\right)\right|}=1
$$

We say that $x_{0} \in J$ is a point of right-hand $\mu$-stat density for the set $M \in \mathscr{B}$ if

$$
\lim _{\delta \rightarrow 0} \frac{\left|M \cap O_{\delta}^{+}\left(x_{0}\right)\right|}{\left|O_{\delta}^{+}\left(x_{0}\right)\right|}=1
$$

By $J_{s t}^{+}\left(x_{0}\right)$ we denote the family of all subsets of $\mathscr{B}$, for which the point $x_{0}$ is a point of righthand $\mu$-stat density.

Similarly, we define the concept of the point of left-hand and right-hand $\mu$-stat density and the family $J_{s t}^{-}\left(x_{0}\right)$.

Assume that the measure $\mu(\cdot)$ additionally satisfies the condition

$$
\text { в) } \lim _{\delta \rightarrow 0} \frac{\left|O_{\delta}^{+}\left(x_{0}\right)\right|}{\left|O_{\delta}^{-}\left(x_{0}\right)\right|}=\lambda \neq 0 .
$$

Let $M \in J_{s t}\left(x_{0}\right)$. Assume $\left|O_{\delta}^{+}\left(x_{0}\right)\right|=\lambda_{\delta}\left|O_{\delta}^{-}\left(x_{0}\right)\right|$. It is clear that $\lambda_{\delta} \rightarrow \lambda$ holds as $\delta \rightarrow 0$. We have

$$
\frac{\left|M \cap O_{\delta}^{0}\left(x_{0}\right)\right|}{\left|O_{\delta}^{0}\left(x_{0}\right)\right|}=\frac{\left|M \cap O_{\delta}^{+}\left(x_{0}\right)\right|}{\left(1+\lambda_{\delta}^{-1}\right)\left|O_{\delta}^{+}\left(x_{0}\right)\right|}+\frac{\left|M \cap O_{\delta}^{-}\left(x_{0}\right)\right|}{\left(1+\lambda_{\delta}\right)\left|O_{\delta}^{-}\left(x_{0}\right)\right|} .
$$

Let

$$
a_{\delta}^{ \pm}=\frac{\left|M \cap O_{\delta}^{ \pm}\left(x_{0}\right)\right|}{\left|O_{\delta}^{ \pm}\left(x_{0}\right)\right|}
$$


We have $0 \leq a_{\delta}^{ \pm} \leq 1, \forall \delta>0$, and

$$
\frac{a_{\delta}^{+}}{1+\lambda_{\delta}^{-1}}+\frac{a_{\delta}^{-}}{1+\lambda_{\delta}} \rightarrow 1, \quad \delta \rightarrow 0
$$

Hence it directly follows that $a_{\delta}^{ \pm} \rightarrow 1$ as $\delta \rightarrow 0$. Indeed, let there exists $\left\{\delta_{n}\right\} \subset(0,+\infty)$ such that $\delta_{n} \rightarrow 0$ and $a_{\delta_{n}}^{+} \rightarrow a<1$ as $n \rightarrow \infty$. Then from (2) we obtain

$$
\frac{a_{\delta_{+}}^{+}}{1+\lambda_{\delta_{n}}^{-1}}+\frac{a_{\delta_{n}}^{-}}{1+\lambda_{\delta_{n}}} \leq \frac{a_{\delta_{n}}}{1+\lambda_{\delta}^{-1}}+\frac{1}{1+\lambda_{\delta_{n}}} \rightarrow \frac{a}{1+\lambda^{-1}}+\frac{1}{1+\lambda}<\frac{1}{1+\lambda^{-1}}+\frac{1}{1+\lambda}=1 .
$$

And this contradicts the relation (2). Thus, if the measure $\mu(\cdot)$ satisfies the condition $\beta$ ), then $M \in J_{s t}\left(x_{0}\right) \Rightarrow M \in J_{\text {st }}^{ \pm}\left(x_{0}\right)$.

Conversely, suppose that

$$
\lim _{\delta \rightarrow 0} \frac{\left|M \cap O_{\delta}^{ \pm}\left(x_{0}\right)\right|}{\left|O_{\delta}^{ \pm}\left(x_{0}\right)\right|}=1
$$

holds. We have

$$
\frac{\left|M \cap O_{\delta}^{0}\left(x_{0}\right)\right|}{\left|O_{\delta}^{0}\left(x_{0}\right)\right|}=\frac{\left|M \cap O_{\delta}^{+}\left(x_{0}\right)\right|}{\left|O_{\delta}^{+}\left(x_{0}\right)\right|} \frac{\left|O_{\delta}^{+}\left(x_{0}\right)\right|}{\left|O_{\delta}^{+}\left(x_{0}\right)\right|+\left|O_{\delta}^{-}\left(x_{0}\right)\right|}+\frac{\left|M \cap O_{\delta}^{-}\left(x_{0}\right)\right|}{\left|O_{\delta}^{-}\left(x_{0}\right)\right|} \frac{\left|O_{\delta}^{-}\left(x_{0}\right)\right|}{\left|O_{\delta}^{+}\left(x_{0}\right)\right|+\left|O_{\delta}^{-}\left(x_{0}\right)\right|} .
$$

If the condition $\beta$ ) is valid, hence we obtain

$$
1 \geq \frac{\left|M \cap O_{\delta}^{0}\left(x_{0}\right)\right|}{\left|O_{\delta}^{0}\left(x_{0}\right)\right|}=\frac{\left|M \cap O_{\delta}^{+}\left(x_{0}\right)\right|}{\left|O_{\delta}^{+}\left(x_{0}\right)\right|} \frac{1}{1+\lambda_{\delta}^{-1}}+\frac{\left|M \cap O_{\delta}^{-}\left(x_{0}\right)\right|}{\left|O_{\delta}^{-}\left(x_{0}\right)\right|} \frac{1}{1+\lambda_{\delta}} \rightarrow \frac{1}{1+\lambda^{-1}}+\frac{1}{1+\lambda}=1
$$

as $\delta \rightarrow 0$. Consequently, $M \in J_{\text {st }}\left(x_{0}\right)$. So, the following proposition is true.

Proposition 1. Let the measure $\mu(\cdot)$ satisfy the conditions $\alpha$ ) and $\beta$ ). Then

$$
M \in J_{s t}\left(x_{0}\right) \Leftrightarrow M \in J_{s t}^{ \pm}\left(x_{0}\right) .
$$

Proceeding from these concepts $\mu$-stat one-sided limits of the function $f(\cdot)$ at the point $x_{0}$ are defined. Namely, we say that the function $f(\cdot)$ has a $\mu$-stat right-hand (left-hand) limit equal to $l$ at a point $x_{0}$ if

$$
\Delta_{\varepsilon}(f ; l) \in J_{s t}^{+}\left(x_{0}\right)\left(\Delta_{\varepsilon}(f ; l) \in J_{s t}^{-}\left(x_{0}\right)\right), \quad \forall \varepsilon>0,
$$

and this fact will be denoted as

$$
\mu \text {-st } \lim _{x \rightarrow x_{0}+0} f(x)=l\left(\mu \text {-st } \lim _{x \rightarrow x_{0}-0} f(x)=l\right) .
$$

Similarly to the case of $\mu$-stat limit, it is proved that these concepts are correct, i.e. if onesided $\mu$-stat limits exist, then they are unique.

It is clear that if $\exists \mu$-st $\lim _{x \rightarrow x_{0}} f(x)$, then $\exists \mu$-st $\lim _{x \rightarrow x_{0} \pm 0} f(x)$ and

$$
\mu \text {-st } \lim _{x \rightarrow x_{0}} f(x)=\mu \text {-st } \lim _{x \rightarrow x_{0}+0} f(x)=\mu \text {-st } \lim _{x \rightarrow x_{0}-0} f(x) \text {. }
$$

Now, let there exist one-sided $\mu$-stat limits and they are equal, i.e.

$$
\mu \text {-st } \lim _{x \rightarrow x_{0}+0} f(x)=\mu \text {-st } \lim _{x \rightarrow x_{0}-0} f(x)=l \text {. }
$$

Take $\forall \varepsilon>0$. We have $\Delta_{\varepsilon}(f ; l) \in J_{s t}^{ \pm}\left(x_{0}\right)$. Then it follows from Proposition 1 that if the condition $\beta)$ is fulfilled, then $\Delta_{\varepsilon}(f ; l) \in J_{s t}\left(x_{0}\right)$. From the arbitrariness of $\Delta_{\varepsilon}(f ; e) \in I_{s t}^{ \pm}\left(x_{0}\right)$, it follows that there exists $\mu$-stat limit at the point $x_{0}$ and $\mu$-st $\lim _{x \rightarrow x_{0}} f(x)=l$. So, it is valid the following assertion. 
Proposition 2. Let the measure $\mu(\cdot)$ satisfy the conditions $\alpha$ ) and $\beta)$. If at the point $x_{0}$ there exist one-sided $\mu$-stat limits, that are equal to $\mu$-stat limit and conversely, then there exists a $\mu$-stat limit of the function $f(\cdot)$ at this point.

Theorem 1. Let the measure $\mu(\cdot)$ satisfy the conditions $\alpha$ ) and $\beta$ ). Then the following statements are equivalent to each other:

$$
\begin{aligned}
& \text { i) } \exists \mu \text {-st } \lim _{x \rightarrow x_{0}} f(x)=l ; \\
& \text { ii) } \exists M \in J_{s t}\left(x_{0}\right): \lim _{\substack{x \rightarrow x_{0} \\
x \in M}} f(x)=l .
\end{aligned}
$$

Proof. Let

$$
M \in J_{s t}\left(x_{0}\right) \wedge \lim _{\substack{x \rightarrow x_{0} \\ x \in M}} f(x)=l
$$

be fulfilled. Take $\forall \varepsilon>0$. Then we have

$$
\exists \delta_{0}>0 \forall \delta \in\left(0, \delta_{0}\right)|f(x)-l|<\varepsilon, \quad \forall x \in M \cap O_{\delta}^{0}\left(x_{0}\right) .
$$

Let $t>0$ be an arbitrary fixed number. Assume

$$
M_{t}\left(x_{0}\right)=M \cap O_{t}^{0}\left(x_{0}\right) \& M_{t}^{c}\left(x_{0}\right)=M \backslash M_{t}\left(x_{0}\right) .
$$

Consequently $M \cap O_{\delta}^{0}\left(x_{0}\right)=\left(M_{t}^{c}\left(x_{0}\right) \cap O_{\delta}^{0}\left(x_{0}\right)\right) \cup\left(M_{t}\left(x_{0}\right) \cap O_{\delta}^{0}\left(x_{0}\right)\right)$, and, as a result

$$
\left|M \cap O_{\delta}^{0}\left(x_{0}\right)\right|=\left|M_{t}^{c}\left(x_{0}\right) \cap O_{\delta}^{0}\left(x_{0}\right)\right|+\left|M_{t}\left(x_{0}\right) \cap O_{\delta}^{0}\left(x_{0}\right)\right| .
$$

It is absolutely obvious that for $\delta<t$ the following $\left(M_{t}\left(x_{0}\right) \cap O_{\delta}^{0}\left(x_{0}\right)\right) \equiv M \cap O_{\delta}^{0}\left(x_{0}\right)$ is true and therefore

$$
\lim _{\delta \rightarrow 0} \frac{\left|M_{t}\left(x_{0}\right) \cap O_{\delta}^{0}\left(x_{0}\right)\right|}{\left|O_{\delta}^{0}\left(x_{0}\right)\right|}=1,
$$

as $M \in J_{s t}\left(x_{0}\right)$. As a result, we obtain that the following inclusion $M_{t}\left(x_{0}\right) \in J_{s t}\left(x_{0}\right)$ is true for all $t>0$. Then it follows from (4) that

$$
\lim _{\delta \rightarrow 0} \frac{\left|M_{t}^{c}\left(x_{0}\right) \cap O_{\delta}^{0}\left(x_{0}\right)\right|}{\left|O_{\delta}^{0}\left(x_{0}\right)\right|}=0, \forall t>0 .
$$

We have $\Delta_{\varepsilon}(f ; l) \supset M_{t}\left(x_{0}\right)$ for all $t \in\left(0, \delta_{0}\right)$. Consequently, from (5) we obtain

$$
\frac{\left|\Delta_{\varepsilon}(f ; l) \cap O_{\delta}^{0}\left(x_{0}\right)\right|}{\left|O_{\delta}^{0}\left(x_{0}\right)\right|} \geq \frac{\left|M_{t}\left(x_{0}\right) \cap O_{\delta}^{0}\left(x_{0}\right)\right|}{\left|O_{\delta}^{0}\left(x_{0}\right)\right|} \rightarrow 1, \delta \rightarrow 0 .
$$

Hence it directly follows that

$$
\lim _{\delta \rightarrow 0} \frac{\left|\Delta_{\varepsilon}(f ; l) \cap O_{\delta}^{0}\left(x_{0}\right)\right|}{\left|O_{\delta}^{0}\left(x_{0}\right)\right|}=1 .
$$

And this in turn means that

$$
\mu \text {-st } \lim _{x \rightarrow x_{0}} f(x)=l .
$$

Thus, if $\exists M \in J_{s t}\left(x_{0}\right)$ such that the relation (3) holds, then there exists $\mu$-stat limit at the point $x_{0}$, and the relation (6) is true. 
Now, to the contrary, assume that the relation (6) is true. Then

$$
\lim _{\delta \rightarrow 0} \frac{\left|\Delta_{\varepsilon}(f ; l) \cap O_{\delta}^{0}\left(x_{0}\right)\right|}{\left|O_{\delta}^{0}\left(x_{0}\right)\right|}=1, \quad \forall \varepsilon>0 .
$$

Let

$$
M_{n}=\left\{x \in J:|f(x)-A|<\frac{1}{n}\right\}, \quad n \in \mathbb{N}, I_{x}^{+}=\left(x_{0}, x\right), \quad \forall x>x_{0},
$$

and assume $M_{n}^{+}=M_{n} \cap\left\{x>x_{0}\right\}$. We have

$$
\lim _{x \rightarrow x_{0}+0} \frac{\left|M_{n}^{+} \cap I_{x}^{+}\right|}{\left|I_{x}^{+}\right|}=1, \quad \forall n \in \mathbb{N} .
$$

Hence it follows that for all $n \in \mathbb{N}$ there exists $x_{n}^{+} \in J \cap\left\{x>x_{0}\right\}$ such that $x_{1}^{+}>x_{2}^{+}>\ldots$, $x_{n}^{+} \rightarrow x_{0}+0$ as $n \rightarrow \infty$ and

$$
\frac{\left|M_{n}^{+} \cap I_{x}^{+}\right|}{\left|I_{x}^{+}\right|} \geq \frac{n^{2}-1}{n^{2}}, \quad \forall x \in\left(x_{0}, x_{n}^{+}\right]
$$

is valid. We have

$$
\frac{\left|\left(M_{n}\right)^{c} \cap I_{x}^{+}\right|}{\left|I_{x}^{+}\right|}=1-\frac{\left|M_{n} \cap I_{x}^{+}\right|}{\left|I_{x}^{+}\right|} \leq 1-\frac{n^{2}-1}{n^{2}}=\frac{1}{n^{2}}, \quad \forall n \in \mathbb{N} .
$$

Denote $M^{+}=\bigcup_{n=1}^{\infty} A_{n}^{+}$, where $A_{n}^{+}=\left[x_{n+1}^{+}, x_{n}^{+}\right) \cap M_{n}^{+}, \forall n \in \mathbb{N}$. Let us show that

$$
\lim _{x \rightarrow x_{0}+0} \frac{\left|M^{+} \cap I_{x}^{+}\right|}{\left|I_{x}^{+}\right|}=1 .
$$

Let $n_{x}=\min \left\{n: x \in\left[x_{n+1}^{+}, x_{n}^{+}\right)\right\}$. We have

$$
\begin{gathered}
M^{+} \cap I_{x}^{+}=\left[M_{n_{x}}^{+} \cap\left[x_{n_{x}+1^{+}}^{+}, x\right)\right] \cup \bigcup_{k=n_{x}+1}^{\infty} A_{k}^{+} . \\
\left|M^{+} \cap I_{x}^{+}\right|=\left|M_{n_{x}}^{+} \cap\left[x_{n_{x}+1}^{+}, x\right)\right|+\sum_{k=n_{x}+1}^{\infty} A_{k}^{+} .
\end{gathered}
$$

Let $k \geq n_{x}+1$ be an arbitrary number. It holds

$$
\begin{gathered}
M_{n_{x}}^{+} \cap\left[x_{k+1}^{+}, x_{k}^{+}\right)=\left[M_{k}^{+} \cap\left[x_{k+1}^{+}, x_{k}^{+}\right)\right] \cup\left[M_{n_{x}}^{+} \cap\left(M_{k}^{+}\right)^{c} \cap\left[x_{k+1}^{+}, x_{k}^{+}\right)\right] \\
=A_{k}^{+} \cup\left[M_{n_{x}}^{+} \cap\left(M_{k}^{+}\right)^{c} \cap\left[x_{k+1}^{+}, x_{k}^{+}\right)\right] . \\
\left|A_{k}^{+}\right|=\left|M_{n_{x}}^{+} \cap\left[x_{k+1}^{+}, x_{k}^{+}\right)\right|-\left|M_{n_{x}}^{+} \cap\left(M_{k}^{+}\right)^{c} \cap\left[x_{k+1}^{+}, x_{k}^{+}\right)\right| .
\end{gathered}
$$

So

$$
\left[\left(M_{k}^{+}\right)^{c} \cap\left[x_{k+1}^{+}, x_{k}^{+}\right)\right] \subset\left[\left(M_{k}^{+}\right)^{c} \cap I_{x_{k}^{+}}^{+}\right] \quad \text { and } \quad\left|I_{x}^{+}\right| \geq\left|I_{x_{k}^{+}}^{+}\right| \Leftrightarrow \frac{1}{\left|I_{x}^{+}\right|} \leq \frac{1}{\left|I_{x_{k}^{+}}^{+}\right|} .
$$


Paying attention to (7), we have

$$
\frac{\left|\left(M_{k}^{+}\right)^{c} \cap\left[x_{k+1}^{+}, x_{k}^{+}\right)\right|}{\left|I_{x}^{+}\right|} \leq \frac{\left|\left(M_{k}^{+}\right)^{c} \cap I_{x_{k}^{+}}^{+}\right|}{\left|I_{x_{k}^{+}}^{+}\right|} \leq \frac{1}{k^{2}} .
$$

Thus

$$
\frac{\left|M_{n_{x}}^{+} \cap\left(M_{k}^{+}\right)^{c} \cap\left[x_{k+1}^{+}, x_{k}^{+}\right)\right|}{\left|I_{x}^{+}\right|} \leq \frac{\left|\left(M_{k}^{+}\right)^{c} \cap\left[x_{k+1}^{+}, x_{k}^{+}\right)\right|}{\left|I_{x}^{+}\right|} \leq \frac{1}{k^{2}}
$$

and, as a result

$$
\frac{\left|A_{k}^{+}\right|}{\left|I_{x}^{+}\right|} \geq \frac{\left|M_{n_{x}}^{+} \cap\left[x_{k+1^{+}}^{+}, x_{k}^{+}\right)\right|}{\left|I_{x}^{+}\right|}-\frac{1}{k^{2}}, \quad \forall k \geq n_{x}+1 .
$$

Then from the relation (9) it follows

$$
\frac{\left|M^{+} \cap I_{x}^{+}\right|}{\left|I_{x}^{+}\right|} \geq \frac{\left|M_{n_{x}}^{+} \cap\left[x_{n_{x}+1^{+}}^{+}, x\right)\right|}{\left|I_{x}^{+}\right|}+\frac{\sum_{k=n_{x}+1}\left|M_{n_{x}}^{+} \cap\left[x_{k+1}^{+}, x_{k}^{+}\right)\right|}{\left|I_{x}^{+}\right|}-\sum_{k=n_{x}+1} \frac{1}{k^{2}} .
$$

It is easy to see that the relation

$$
\left(M_{n_{x}}^{+} \cap I_{x}^{+}\right)=\left[M_{n_{x}}^{+} \cap\left[x_{n_{x}+1}^{+}, x\right)\right] \cup \bigcup_{k=n_{x}+1}^{\infty}\left[M_{n_{x}}^{+} \cap\left[x_{k+1}^{+}, x_{k}^{+}\right)\right],
$$

is valid. Then from the previous inequality we have

$$
\frac{\left|M^{+} \cap I_{x}^{+}\right|}{\left|I_{x}^{+}\right|} \geq \frac{\left|M_{n_{x}}^{+} \cap I_{x}^{+}\right|}{\left|I_{x}^{+}\right|}-\sum_{k=n_{x}+1} \frac{1}{k^{2}} .
$$

From (7) it follows

$$
\frac{\left|M^{+} \cap I_{x}^{+}\right|}{\left|I_{x}^{+}\right|} \geq \frac{n_{x}^{2}-1}{n_{x}^{2}}-\sum_{k=n_{x}+1} \frac{1}{k^{2}} .
$$

It is absolutely clear that from $x \rightarrow x_{0}+0$ follows $n_{x} \rightarrow \infty$. Then from (10) we have

$$
1 \geq \lim _{x \rightarrow \infty} \frac{\left|M^{+} \cap I_{x}^{+}\right|}{\left|I_{x}^{+}\right|} \geq \lim _{n_{x} \rightarrow \infty} \frac{n_{x}^{2}-1}{n_{x}^{2}}-\lim _{n_{x} \rightarrow \infty} \sum_{k=n_{x}+1} \frac{1}{k^{2}}=1 .
$$

Hence, it is proved that $M \in J_{s t}^{+}\left(x_{0}\right)$.

Let us show that $\lim _{\substack{x \rightarrow x_{0}+0 \\ x \in M^{+}}} f(x)=A$. Let $\varepsilon>0$ be an arbitrary number. Let $n_{0} \in \mathbb{N}$ be such that $\frac{1}{n_{0}}<\varepsilon$. Then for all $x \in\left(x_{0}, \frac{1}{n_{0}}\right)$ we have

$$
\left(M^{+} \cap I_{x}^{+}\right) \subset\left(M_{n_{0}}^{+} \cap I_{x}^{+}\right) \Rightarrow|f(y)-A|<\frac{1}{n_{0}}<\varepsilon, \quad \forall y \in\left(M^{+} \cap I_{x}^{+}\right),
$$

that is $\lim _{\substack{x \rightarrow x_{0}+0 \\ x \in M^{+}}} f(x)=A$. Thus, if the measure $\mu(\cdot)$ satisfies the condition $\beta$ ) and (6) holds, then

$$
\exists M^{+} \in J_{s t}^{+}\left(x_{0}\right): \lim _{\substack{x \rightarrow x_{0}+0 \\ x \in M^{+}}} f(x)=l .
$$


In the same way we prove that if the measure $\mu(\cdot)$ satisfies the condition $\beta$ ) and (6) holds, then

$$
\exists M^{-} \in J_{s t}^{-}\left(x_{0}\right): \lim _{\substack{x \rightarrow x_{0}-0 \\ x \in M^{-}}} f(x)=l .
$$

Let $M=M^{-} \cup M^{+}$. Proposition 1 implies $M \in J_{s t}\left(x_{0}\right)$ and it is clear that $\lim _{\substack{x \rightarrow x_{0} \\ x \in M}} f(x)=l$.

Following the works $[7,16,38]$, let us define the next concept.

Definition 3. We say that $s t \lim _{n \rightarrow \infty} a_{n}=a$, if

$$
\lim _{n \rightarrow \infty} \frac{\sum_{k=1}^{n} \chi_{a(\varepsilon)}(k)}{n}=1, \quad \forall \varepsilon>0,
$$

where $a(\varepsilon) \equiv\left\{k \in \mathbb{N}:\left|a_{k}-a\right| \geq \varepsilon\right\}, \chi_{M}(\cdot)$ is the characteristic function of the set $M$.

Definition 4. We say that the function $f: J \rightarrow \mathbb{R}$ has a statistical limit $A$ at the point $a \in J$, if st $\lim _{n \rightarrow \infty} f\left(a_{n}\right)=A$ for all $\left\{a_{n}\right\}_{n \in N} \subset J$ such that st $\lim _{n \rightarrow \infty} a_{n}=a$. This fact will be denoted as st $\lim _{x \rightarrow a} f(x)=A$.

Theorem 2. Let the measure $\mu(\cdot)$ satisfy the condition $\alpha)$. Then if $\exists$ st $\lim _{x \rightarrow a} f(x)$, then $\exists \mu$-st $\lim _{x \rightarrow a} f(x)$ and they are equal. The converse is generally not true.

Proof. Consider the following function

$$
f(x)=\left\{\begin{array}{l}
n, x=\frac{1}{n}, \\
x, x \in[-1,1] \backslash\left\{\frac{1}{n}\right\}_{n \in \mathbb{N}} .
\end{array}\right.
$$

As $\mu(\cdot)$ we take a Lebesgue measure on $[-1,1]$. It is easy to see that $\mu$-st $\lim _{x \rightarrow 0} f(x)=0$, in this case st $\lim _{x \rightarrow a} f(x)$ does not exist. This example shows that the Definitions 1 and 2 are not equivalent, from the first definition does not follow the second.

Now, to the contrary, assume that $\exists$ st $\lim _{x \rightarrow a} f(x)=A$. Let the relation $\mu$-st $\lim _{x \rightarrow a} f(x)=A$ does not hold. Consequently, $\exists \varepsilon_{0}>0$ such that the relation

$$
\lim _{\delta \rightarrow 0} \frac{\left|\Delta_{\varepsilon_{0}}^{c}(f ; A) \cap O_{\delta}^{0}(a)\right|}{\left|O_{\delta}^{0}(a)\right|}=0
$$

does not hold, where $\Delta_{\varepsilon_{0}}^{c}(f ; A)=J \backslash \Delta_{\varepsilon_{0}}(f ; A)=\left\{x \in J:|f(x)-A| \geq \varepsilon_{0}\right\}$. Thus there exist $\delta_{0}>0$ and $\left\{\delta_{n}\right\}_{n \in \mathbb{N}}$ such that $\delta_{1}>\delta_{2}>\ldots, \delta_{n} \rightarrow 0$, and

$$
\begin{gathered}
\frac{\left|\Delta_{\varepsilon_{0}}^{c}(f ; A) \cap O_{\delta_{n}}^{0}(a)\right|}{\left|O_{\delta_{n}}^{0}(a)\right|} \geq \delta_{0}, \quad \text { i.e. } \\
\left|\Delta_{\varepsilon_{0}}^{c}(f ; A) \cap O_{\delta_{n}}^{0}(a)\right| \geq \delta_{0}\left|O_{\delta_{n}}^{0}(a)\right|>0, \quad \forall n \in \mathbb{N} .
\end{gathered}
$$

It follows directly from the condition $\alpha)$ that $\left|\Delta_{\varepsilon_{0}}^{c}(f ; A) \cap O_{\delta_{n}}^{0}(a)\right| \rightarrow 0$ as $n \rightarrow \infty$.

Then from (11) we obtain

$$
\exists\left\{a_{k}\right\}_{k \in N}: a_{k} \in \Delta_{\varepsilon_{0}}^{c}(f ; A) \cap O_{\delta_{n_{k}}}^{0}(a) \wedge a_{k} \in O_{\delta_{n_{k}}}^{0}(a) \backslash O_{\delta_{n_{k+1}}}^{0}(a) .
$$

It is obvious that $\lim _{k \rightarrow \infty} a_{k}=a$, but on the other hand $\left|f\left(a_{k}\right)-A\right| \geq \varepsilon_{0}$ for all $k \in \mathbb{N}$. Thus, the relation st $\lim _{n \rightarrow \infty} f\left(a_{n}\right)=A$ is not true. The resulting contradiction proves the theorem. 


\section{$2 \mu$-stat fundamentality}

Let us define the concept of $\mu$-statistical fundamentality.

Definition 5. We say that the function $f: J \rightarrow \mathbb{R}$ is fundamental at a point $a \in J$ if for any $\varepsilon>0$ there exists $x_{\varepsilon} \in J$ such that

$$
\lim _{\delta \rightarrow 0} \frac{\left|\Delta\left(f ; x_{\varepsilon}\right) \cap O_{\delta}^{0}(a)\right|}{\left|O_{\delta}^{0}(a)\right|}=1,
$$

where $\Delta\left(f ; x_{\varepsilon}\right)=\left\{x \in J:\left|f(x)-f\left(x_{\varepsilon}\right)\right|<\varepsilon\right\}$.

Assume that the measure $\mu(\cdot)$ satisfies the conditions $\alpha), \beta)$ and $\exists \mu$-st $\lim _{x \rightarrow a} f(x)=A$. Then by Theorem 1

$$
\exists M \in J_{s t}(a): \lim _{M \ni x \rightarrow a} f(x)=A .
$$

Hence, we obtain that for all $\varepsilon>0$ there exists $\delta_{\varepsilon}>0$ such that

$$
|f(x)-f(y)|<\varepsilon, \quad \forall x, y \in M \cap O_{\delta}^{0}(a), \forall \delta \leq \delta_{\varepsilon} .
$$

Take $\forall x_{\varepsilon} \in M \cap O_{\delta_{\varepsilon}}^{0}(a)$. We have $\left|f(x)-f\left(x_{\varepsilon}\right)\right|<\varepsilon$ for all $x \in M \cap O_{\delta}^{0}(a)$ and $\delta \leq \delta_{\varepsilon}$.

Consequently $\left(M \cap O_{\delta}^{0}(a)\right) \subset \Delta\left(f ; x_{\varepsilon}\right)$, and, as a result

$$
\frac{\left|M \cap O_{\delta}^{0}(a)\right|}{\left|O_{\delta}^{0}(a)\right|} \leq \frac{\left|\Delta\left(f ; x_{\varepsilon}\right) \cap O_{\delta}^{0}(a)\right|}{\left|O_{\delta}^{0}(a)\right|}, \forall \delta \leq \delta_{\varepsilon} .
$$

From $M \in J_{s t}(a)$ it follows that

$$
\lim _{\delta \rightarrow 0} \frac{\left|M \cap O_{\delta}^{0}(a)\right|}{\left|O_{\delta}^{0}(a)\right|}=1 .
$$

Then from (12) we obtain that

$$
\lim _{\delta \rightarrow 0} \frac{\left|\Delta\left(f ; x_{\varepsilon}\right) \cap O_{\delta}^{0}(a)\right|}{\left|O_{\delta}^{0}(a)\right|}=1 .
$$

We will also need the following lemma.

Lemma 1. Let $M_{k} \in J_{s t}(a), k=1,2$, then $M_{1} \cap M_{2} \in J_{s t}(a)$.

Proof. We have $M_{1} \cap M_{2}=\left(M_{1} \cup M_{2}\right) \backslash\left(M_{1} \Delta M_{2}\right)$, where $M_{1} \Delta M_{2}=\left(M_{2} \backslash M_{1}\right) \cup\left(M_{1} \backslash M_{2}\right)$ is a symmetric difference of sets $M_{1}$ and $M_{2}$. Consequently,

$$
M_{1} \cap M_{2} \cap O_{\delta}^{0}(a)=\left[\left(M_{1} \cup M_{2}\right) \cap O_{\delta}^{0}(a)\right] \backslash\left[\left(M_{1} \Delta M_{2}\right) \cap O_{\delta}^{0}(a)\right] .
$$

We have

$$
\left(M_{1} \Delta M_{2}\right) \cap O_{\delta}^{0}(a)=\left[\left(M_{2} \backslash M_{1}\right) \cap O_{\delta}^{0}(a)\right] \cup\left[\left(M_{1} \backslash M_{2}\right) \cap O_{\delta}^{0}(a)\right] .
$$

We pay attention to the fact that

$$
\left(M_{2} \backslash M_{1}\right) \cap O_{\delta}^{0}(a)=M_{1}^{c} \cap O_{\delta}^{0}(a),
$$


where $M^{c}=J \backslash M$ is a complement of a set $M$ in $J$. From $M_{1} \in J_{s t}(a)$ it follows that

$$
\lim _{\delta \rightarrow 0} \frac{\left|M_{1}^{c} \cap O_{\delta}^{0}(a)\right|}{\left|O_{\delta}^{0}(a)\right|}=0 .
$$

Then from (14) we obtain $\frac{\left|\left(M_{2} \backslash M_{1}\right) \cap O_{\delta}^{0}(a)\right|}{\left|O_{\delta}^{0}(a)\right|} \rightarrow 0, \delta \rightarrow 0$. Similarly we establish $\frac{\left|\left(M_{1} \backslash M_{2}\right) \cap O_{\delta}^{0}(a)\right|}{\left|O_{\delta}^{0}(a)\right|} \rightarrow 0$, $\delta \rightarrow 0$. Thus, it is valid

It is obvious that

$$
\frac{\left|\left(M_{1} \Delta M_{2}\right) \cap O_{\delta}^{0}(a)\right|}{\left|O_{\delta}^{0}(a)\right|} \rightarrow 0, \quad \delta \rightarrow 0
$$

$$
\frac{\left|\left(M_{1} \cup M_{2}\right) \cap O_{\delta}^{0}(a)\right|}{\left|O_{\delta}^{0}(a)\right|} \rightarrow 1, \quad \delta \rightarrow 0 .
$$

From (13) we directly obtain $\frac{\left|\left(M_{1} \cap M_{2}\right) \cap O_{\delta}^{0}(a)\right|}{\left|O_{\delta}^{0}(a)\right|}=\frac{\left|\left(M_{1} \cup M_{2}\right) \cap O_{\delta}^{0}(a)\right|}{\left|O_{\delta}^{0}(a)\right|}-\frac{\left|\left(M_{1} \Delta M_{2}\right) \cap O_{\delta}^{0}(a)\right|}{\left|O_{\delta}^{0}(a)\right|}$. Taking into account (14) and (15) we have

$$
\frac{\left|\left(M_{1} \cap M_{2}\right) \cap O_{\delta}^{0}(a)\right|}{\left|O_{\delta}^{0}(a)\right|} \rightarrow 1, \quad \delta \rightarrow 0, \quad \text { i.e. } \quad M_{1} \cap M_{2} \in J_{s t}(a) .
$$

The lemma is proved.

Theorem 3. Let the measure $\mu(\cdot)$ satisfies the conditions $\alpha$ ) and $\beta)$. Then the function $f: J \rightarrow \mathbb{R}$ is $\mu$-stat fundamental at a point $a \in R$ if and only if $\exists \mu$-st $\lim _{x \rightarrow a} f(x)$.

Proof. Let us assume that the function $f: J \rightarrow \mathbb{R}$ is a $\mu$-statistical fundamental at a point $a \in J$. Then for $\varepsilon_{1}=1$ there exists $x_{1} \in J$ such that

$$
\lim _{\delta \rightarrow 0} \frac{\left|\Delta\left(f ; x_{1}\right) \cap O_{\delta}^{0}(a)\right|}{\left|O_{\delta}^{0}(a)\right|}=1,
$$

where $\Delta\left(f ; x_{k}\right)=\left\{x \in J:\left|f(x)-f\left(x_{k}\right)\right|<\varepsilon_{k}\right\}, k \in \mathbb{N}$. Consequently, $\Delta\left(f ; x_{1}\right) \in J_{s t}(a)$. Similarly, for $\varepsilon_{2}=\frac{1}{2}$ there exists $x_{2} \in J$ such that $\Delta\left(f ; x_{2}\right) \in J_{s t}(a)$. By Lemma 1 , we obtain

$$
\Delta\left(f ; x_{1}\right) \cap \Delta\left(f ; x_{2}\right) \equiv J_{1} \in J_{s t}(a) .
$$

Let

$$
R_{J_{1}}=\left\{f(x): x \in J_{1}\right\} \quad \text { and } \quad I_{2} \equiv\left[f\left(x_{2}\right)-\frac{1}{2}, f\left(x_{2}\right)+\frac{1}{2}\right] .
$$

It is clear that $R_{J_{1}} \subset I_{2}$.

Similarly, we define $\Delta\left(f ; x_{4}\right) \equiv\left\{x \in J:\left|f(x)-f\left(x_{4}\right)\right|<\frac{1}{4}\right\}$, and consider $J_{2} \equiv J_{1} \cap \Delta\left(f ; x_{4}\right)$. Again, by Lemma 1, we have $J_{2} \in J_{s t}(a)$. Put $R_{J_{2}}=\left\{f(x): x \in J_{2}\right\}$. Let

$$
R_{J_{2}} \subset I_{2^{2}} \equiv\left(\left[f\left(x_{4}\right)-\frac{1}{4}, f\left(x_{4}\right)+\frac{1}{4}\right] \cap I_{2}\right) .
$$

Continuing this process, we obtain a sequence of segments $I_{2^{n}}$ and sets $R_{J_{n}} \subset I_{2^{n}}$, with the following properties

$$
\begin{aligned}
& I_{2^{1}} \supset I_{2^{2}} \supset \ldots, \quad d\left(I_{2^{n}}\right) \leq \frac{1}{2^{n-1}}, \\
& R_{J_{n}} \equiv\left\{f(x): x \in J_{n}\right\} \subset I_{2^{n}}, \\
& J_{n-1} \equiv \Delta\left(f ; x_{n-1}\right) \cap \Delta\left(f ; x_{n}\right), \\
& J_{n} \in J_{s t}(a), \quad \forall n \in \mathbb{N},
\end{aligned}
$$


where $d(I)$ is the length of the segment $I$.

Absolutely obvious that $\exists ! A \in \bigcap_{n=1}^{\infty} I_{2^{n}}$.

Let us show that $\mu$-st $\lim _{x \rightarrow a} f(x)=A$. Let $\varepsilon>0$ be an arbitrary number. It is clear that there exists $n_{0} \in \mathbb{N}$ such that

$$
I_{2^{n}} \subset\left(A-\frac{\varepsilon}{2}, A+\frac{\varepsilon}{2}\right), \quad \forall n \geq n_{0} .
$$

Thus, we have

$$
R_{J_{n_{0}}} \subset I_{2^{n_{0}}} \equiv\left[f\left(x_{2^{n_{0}}}\right)-\frac{1}{2^{n_{0}}}, f\left(x_{2^{n_{0}}}\right)+\frac{1}{2^{n_{0}}}\right] \cap I_{2^{n_{0}-1}} .
$$

On the other hand $R_{J_{n_{0}}} \equiv\left\{f(x): x \in J_{n_{0}}\right\}$, and, due to the structure of $J_{n_{0}} \in J_{s t}(a)$, where $J_{n_{0}} \equiv J_{n_{0}-1} \cap \Delta\left(f ; x_{2^{n_{0}}}\right)$, we have

$$
\Delta\left(f ; x_{2^{n_{0}}}\right) \equiv\left\{x \in J:\left|f(x)-f\left(x_{2^{n_{0}}}\right)\right|<\frac{1}{2^{n_{0}}}\right\} .
$$

Choose $n_{0}$ from the condition $\frac{1}{2^{n_{0}}}<\frac{\varepsilon}{2}$. We have

$$
|f(x)-A| \leq\left|f(x)-f\left(x_{2^{n_{0}}}\right)\right|+\left|f\left(x_{2^{n_{0}}}\right)-A\right|<\left|f(x)-f\left(x_{2^{n_{0}}}\right)\right|+\frac{\varepsilon}{2} .
$$

Hence it directly follows that

$$
\left\{x \in J:\left|f(x)-f\left(x_{2^{n_{0}}}\right)\right|<\frac{1}{2^{n_{0}}}\right\} \subset\{x \in J:|f(x)-A|<\varepsilon\}, \quad \text { i.e. } \quad \Delta\left(f ; x_{2^{n_{0}}}\right) \subset \Delta_{\varepsilon}(f ; A) .
$$

Since, $\Delta\left(f ; x_{2^{n}}\right) \in J_{s t}(a)$, from the previous inclusion follows that $\Delta_{\varepsilon}(f ; A) \in J_{s t}(a)$. From the arbitrariness of $\varepsilon>0$, we obtain $\mu$-st $\lim _{x \rightarrow a} f(x)=A$. Thus, the theorem is proved.

Definition 6. The functions $f ; g: J \rightarrow \mathbb{R}$ are called $\mu$-statistical equivalent at a point $a \in J$ if $J_{f ; g} \in J_{s t}(a)$, where

$$
J_{f ; g} \equiv\{x \in J: f(x)=g(x)\} .
$$

This fact will be denoted as $f \stackrel{s t}{\sim} g, x \rightarrow a$.

Assume that $\exists \mu$-st $\lim _{x \rightarrow a} f(x)=A$. Then by Theorem 1, there exists $M \in J_{s t}(a)$ such that $\lim _{M \ni x \rightarrow a} f(x)=A$. Define

$$
g(x) \equiv\left\{\begin{array}{l}
f(x), x \in M \\
A, x \in M^{c}
\end{array}\right.
$$

It is clear that $M \subset J_{f ; g} \Rightarrow J_{f ; g} \in J_{s t}(a) \Rightarrow f \stackrel{s t}{\sim} g, x \rightarrow a$. Clearly, $\lim _{x \rightarrow a} g(x)=A$.

Vice versa, let

$$
\lim _{x \rightarrow a} g(x)=A \wedge f \stackrel{s t}{\sim} g, x \rightarrow a .
$$

Then it is easy to see that $\mu$-st $\lim _{x \rightarrow a} f(x)=A$. As a result, the following assertion is valid.

Theorem 4. Let the measure $\mu(\cdot)$ satisfies the conditions $\alpha$ ) and $\beta$ ). Then for the function $f: J \rightarrow \mathbb{R}$ the following statements are equivalent to each other:

1) $\exists \mu$-st $\lim _{x \rightarrow a} f(x)$,

2) $f$ is $\mu$-stat fundamental at the point $x=a$,

3) $\exists g: J \rightarrow \mathbb{R} \wedge \exists \lim _{x \rightarrow a} g(x) \wedge f \stackrel{s t}{\sim} g, x \rightarrow a$. 


\section{The space of $\mu$-stat continuous functions}

Similar to the classical case, if

$$
\mu \text {-st } \lim _{x \rightarrow x_{0}-0} f(x)=\mu \text {-st } \lim _{x \rightarrow x_{0}+0} f(x) \neq f\left(x_{0}\right),
$$

then $x_{0}$ is called $\mu$-stat removable discontinuity point. If $\exists \mu$-st $\lim _{x \rightarrow x_{0} \pm 0} f(x)$ and

$$
\mu \text {-st } \lim _{x \rightarrow x_{0}-0} f(x) \neq \mu \text {-st } \lim _{x \rightarrow x_{0}+0} f(x),
$$

then $x_{0}$ is called $\mu$-stat discontinuity of the first kind and the quantity

$$
\Delta_{f}^{s t}\left(x_{0}\right)=\mu \text {-st } \lim _{x \rightarrow x_{0}+0} f(x)-\mu \text {-st } \lim _{x \rightarrow x_{0}-0} f(x)
$$

is called a $\mu$-stat jump of the function $f$ at $x_{0}$.

In other cases, $x_{0}$ is called a $\mu$-stat discontinuity point of the second kind.

Example 1. Let $(\mathbb{R} ; \mathscr{B} ; \mu)$ be a measurable space with a Lebesgue measure. Consider the function

$$
f(x)= \begin{cases}\sin x, & x \in \mathbb{Q}, \\ \operatorname{sign} x, & x \in \mathbb{R} \backslash \mathbb{Q},\end{cases}
$$

where $\mathbb{Q}$ are rational numbers in $\mathbb{R}$. The point $x_{0}=0$ is a $\mu$-stat discontinuity of the first kind and $\Delta_{f}^{s t}(0)=2$. All other points are $\mu$-stat continuity points.

If

$$
\mu \text {-st } \lim _{x \rightarrow x_{0}-0} f(x)=\mu \text {-st } \lim _{x \rightarrow x_{0}+0} f(x)=f\left(x_{0}\right),
$$

holds, then $f(\cdot)$ is called a $\mu$-stat continuous at the point $x_{0}$.

Let $f:[a, b] \rightarrow \mathbb{R}$ be some function. It is clear that if $f \in C[a, b]$, then $f(\cdot)$ is a $\mu$-stat continuous on $[a, b]$. The following question arises naturally.

Question 1. Let $f:[a, b] \rightarrow \mathbb{R}$ be a $\mu$-stat continuous on $[a, b]$. Is it continuous on $[a, b]$ ?

It is obvious that if $f(\cdot)$ has a discontinuity of the first kind at the point $x_{0} \in(a, b)$, then $x_{0}$ is also a $\mu$-stat discontinuity point of the first kind and moreover $\mu$-st $f\left(x_{0} \pm 0\right)=f\left(x_{0} \pm 0\right)$. Therefore, if $f(\cdot)$ has a discontinuity of the first kind at the point $x_{0}$, then it can not be a $\mu$-stat continuous at this point.

Denote the linear space of $\mu$-stat continuous functions on $[a, b]$ over the field $\mathbb{K}(\mathbb{K} \equiv \mathbb{C}$ or $\mathbb{R})$ by $C_{s t}[a, b]$. It is absolutely clear that the pointwise limit of the sequence of $\mu$-stat continuous functions may not be $\mu$-stat continuous on $[a, b]$.

Let us give an example of a function on the interval $E=[-1,1]$, which is not continuous on $E$, but at the same time, is $\mu$-statistical continuous on $E$.

Lemma 2. The strict embedding $C[a, b] \subset C_{s t}[a, b], C_{s t}[a, b] \backslash C[a, b] \neq \varnothing$, holds true.

Proof. The embedding $C[a, b] \subset C_{s t}[a, b]$ is obvious. So, we will prove the validity of $C_{s t}[a, b] \backslash C[a, b] \neq \varnothing$. Consider the following series

$$
\sum_{k=1}^{\infty} \alpha_{k}, \quad \alpha_{k}>0, \forall k \in \mathbb{N}
$$


such that the remainder terms satisfy the conditions

$$
\sigma_{n} \leq \frac{1}{(n+1)^{3}}
$$

where $\sigma_{n}=\sum_{k=n}^{\infty} \alpha_{k}$.

As $[a, b]$ we take $[-1,1]$. Let $\mu$ be a Lebesgue measure and $O_{\delta}(x) \equiv(x-\delta, x+\delta) \cap[-1,1]$. Denote by $i_{n} \subset\left(\frac{1}{n+1}, \frac{1}{n}\right)$ an arbitrary interval of length $\alpha_{n}$, i.e. $\left|i_{n}\right|=\mu\left(i_{n}\right)=\alpha_{n}, n \in \mathbb{N}$.

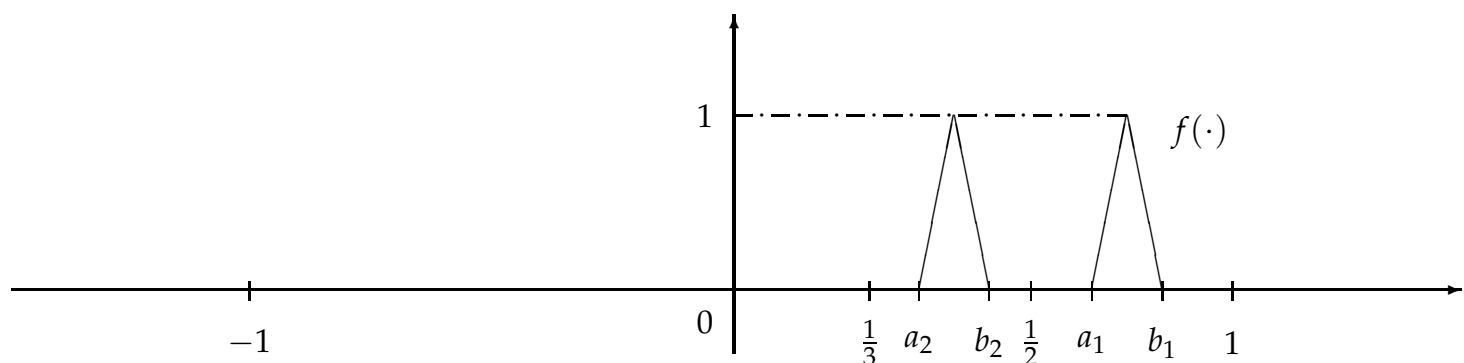

Figure 1

Let $x_{n} \in i_{n}$ be the middle of the interval $i_{n}=\left(a_{n}, b_{n}\right)$. Consider the points $(1 ; 0),\left(b_{1} ; 0\right)$, $\left(x_{1} ; 1\right),\left(a_{1} ; 0\right),\left(b_{2} ; 0\right), \ldots$, and connect them with the broken lines (see Figure 1$)$. Denote the function generated by this graph and the interval $[-1,0]$ by $f(x)$. It is clear that $f \notin C[-1,1]$, because there does not exist $f(+0)$. Let us show that $f \in C_{s t}[-1,1]$. Obviously, $f(\cdot)$ is continuous at every point $x_{0} \neq 0$, and therefore it is $\mu$-stat continuous at these points. Let us show that $f(\cdot)$ is $\mu$-stat continuous at the point $x=0$ too. To do so, it suffices to show that there exist one-sided statistical limits at the point $x=0$ and they are equal to each other.

Let $\varepsilon>0$ be an arbitrary number. It is sufficient to prove that

$$
S_{n}(\varepsilon)=\frac{\left|\{x:|f(x) \geq \varepsilon|\} \cap O_{\frac{1}{n}}(0)\right|}{\left|O_{\frac{1}{n}}(0)\right|} \rightarrow 0, \quad n \rightarrow \infty,
$$

where $|\{\cdot\}|$ is a Lebesgue measure of the set $\{\cdot\}$. So, it is easy to see that

$$
\left(\{x:|f(x) \geq \varepsilon|\} \cap O_{\frac{1}{n}}(0)\right) \subset \bigcup_{k=n}^{\infty} i_{k}
$$

and therefore

$$
\left|\{x:|f(x)| \geq \varepsilon\} \cap O_{\frac{1}{n}}(0)\right| \leq\left|\bigcup_{k=n}^{\infty} i_{k}\right|=\sum_{k=n}^{\infty}\left|i_{k}\right|=\sigma_{n} \leq \frac{1}{(n+1)^{3}} .
$$

Consequently

$$
S_{n}(\varepsilon) \leq \frac{n}{2(n+1)^{3}} \rightarrow 0, \quad n \rightarrow \infty .
$$

This immediately implies that

$$
S_{\delta}(\varepsilon)=\frac{\left|\{x:|f(x)| \geq \varepsilon\} \cap O_{\delta}(0)\right|}{\left|O_{\delta}(0)\right|} \rightarrow 0, \quad \delta \rightarrow 0,
$$

and, as a result $f(\cdot)$ is a $\mu$-stat continuous at $x=0$ and hence $f \in C_{s t}[-1,1]$. The lemma is proved. 
Similarly, we can give an example of non-bounded function on the interval $[-1,1]$, which is a $\mu$-stat continuous on $[-1,1]$.

Lemma 3. The relations $C_{s t}[a, b] \backslash L_{p}(a, b) \neq \varnothing$ and $L_{p}(a, b) \backslash C_{s t}[a, b] \neq \varnothing, \forall p \in[1,+\infty)$, hold true.

Proof. The relation $L_{p}(a, b) \backslash C_{s t}[a, b] \neq \varnothing$ is obvious, since the function having a removable discontinuity point does not belong to $C_{s t}[a, b]$. Let us prove $C_{s t}[a, b] \backslash L_{p}(a, b) \neq \varnothing$.

Consider the series (16), satisfying the condition (17). Similarly to the previous case, we consider the intervals

$$
i_{n}=\left(a_{n}, b_{n}\right) \subset\left(\frac{1}{n+1}, \frac{1}{n}\right):\left|i_{n}\right|=\alpha_{n},
$$

and let $x_{n}=\frac{a_{n}+b_{n}}{2}$. Consider the points $(1 ; 0),\left(b_{1} ; 0\right),\left(x_{1} ; \alpha_{1}^{-1}\right),\left(a_{1} ; 0\right),\left(b_{2} ; 0\right),\left(x_{2} ; \alpha_{2}^{-1}\right), \ldots$ Let us connect them by segments. Denote by $f(x)$ the function obtained by these segments and the segment $[-1,0]$. From previous arguments it follows that $f \in C_{s t}[-1,1]$. We have

$$
\int_{-1}^{1}|f(x)| d x=\sum_{k=1}^{\infty} \int_{i_{k}}|f(x)| d x=\sum_{k=1}^{\infty} \frac{1}{2} \alpha_{k} f\left(x_{k}\right)=\frac{1}{2} \sum_{k=1}^{\infty} 1=+\infty .
$$

Thus, $f \notin L_{p}(0,1), \forall p \in[1,+\infty)$. It is obvious that

$$
C[a, b] \subset\left(C_{s t}[a, b] \cap L_{p}(a, b)\right), \forall p \in[1,+\infty) .
$$

The lemma is proved.

The previous example shows that $C[a, b]$ is not dense in $C_{s t}[a, b]$ with respect to the norm $\|\cdot\|_{p}$. The following question arises naturally.

Question 2. Is there such a metric or such convergence, with respect to which the space $C_{s t}[a, b]$ is complete?

Let

$$
C_{s t}^{J}[a, b] \equiv\left\{f \in C_{s t}[a, b]:\|f\|_{\infty}<+\infty\right\}, \quad \text { where } \quad\|f\|_{\infty}=\sup _{[a, b]}|f(\cdot)| .
$$

It is clear that the following strict embeddings hold true

$$
C[a, b] \subset C_{s t}^{J}[a, b] \subset L_{p}(a, b), \forall p \in(0,+\infty) .
$$

Under $L_{p}(a, b)$ we understand the space of measurable (with respect to the Lebesgue measure) functions on $(a, b)$, for $p \in(0,1)$, with finite integral

$$
\int_{a}^{b}|f(t)|^{p} d t<+\infty
$$

Theorem 5. Let $(\mathbb{R} ; \mathscr{B} ; \mu)$ be a measurable space with a $\sigma$-finite measure $\mu$ on the $\sigma$-algebra of Borel sets $\mathscr{B}$ and

$$
\mu\left(\left(-\infty, x_{0}\right)\right)=\mu\left(\left(x_{0},+\infty\right)\right)=+\infty
$$

for some $x_{0} \in \mathbb{R}$. Then the embeddings:

i) $C[a, b] \subset\left(C_{s t}[a, b] \cap L_{p}(a, b)\right), \forall p \in(0,+\infty)$,

ii) $C[a, b] \subset\left(C_{s t}^{J}[a, b] \subset L_{p}(a, b)\right), \forall p \in(0,+\infty)$,

hold true, and they are strict. 
Theorem 6. The space $C_{s t}^{J}[a, b]$ is a Banach space with respect to the norm $\|\cdot\|_{\infty}$.

Proof. Let us show that the space $C_{s t}^{J}[a, b]$ is complete with respect to the norm of $C[a, b] \subset$ $\left(C_{s t}[a, b] \cap L_{p}(a, b)\right), \forall p \in(0,+\infty)$. Let $\left\{f_{n}\right\}_{n \in \mathbb{N}} \subset C_{s t}^{J}[a, b]$ be some fundamental sequence, i.e. $\left\|f_{n}-f_{m}\right\|_{\infty} \rightarrow 0$ as $n, m \rightarrow \infty$.

Fixing $\forall x \in[a, b]$, we obtain that $\left\{f_{n}(x)\right\}_{n \in \mathbb{N}}$ is a fundamental sequence and, as a result, it converges to a certain value $f(x)$. Let us show that $f \in C_{s t}^{J}[a, b]$. Let $\varepsilon>0$ be an arbitrary number and $x_{0} \in[a, b]$ be an arbitrary point. Take $\forall n \in N$ and let

$$
E_{n}(\varepsilon) \equiv\left\{x:\left|f_{n}(x)-f_{n}\left(x_{0}\right)\right| \geq \frac{\varepsilon}{3}\right\}, \quad E_{n}(f ; \varepsilon) \equiv\left\{x:\left|f(x)-f_{n}(x)\right| \geq \frac{\varepsilon}{3}\right\} .
$$

We have

$$
\left|f(x)-f\left(x_{0}\right)\right| \leq\left|f(x)-f_{n}(x)\right|+\left|f_{n}(x)-f_{n}\left(x_{0}\right)\right|+\left|f_{n}\left(x_{0}\right)-f\left(x_{0}\right)\right| .
$$

It is obvious that $\left\|f_{n}-f\right\|_{\infty} \rightarrow 0$ as $n \rightarrow \infty$. Therefore, it is clear that

$$
\left|f_{n}(x)-f(x)\right|<\frac{\varepsilon}{3}, \forall x \in[a, b] .
$$

Then from (18) it follows that $\left\{x:\left|f(x)-f\left(x_{0}\right)\right| \geq \varepsilon\right\} \subset E_{n}(\varepsilon), \forall n \geq n_{\varepsilon}$. Since, otherwise

$$
\left|f(x)-f\left(x_{0}\right)\right| \leq \frac{2}{3} \varepsilon+\left|f_{n}(x)-f_{n}\left(x_{0}\right)\right|<\frac{2}{3} \varepsilon+\frac{1}{3} \varepsilon=\varepsilon .
$$

Consequently $\left(\left\{x:\left|f(x)-f\left(x_{0}\right)\right| \geq \varepsilon\right\} \cap O_{\delta}\left(x_{0}\right)\right) \subset\left(E_{n}(\varepsilon) \cap O_{\delta}\left(x_{0}\right)\right)$, and, as a result

$$
\left|\left\{x:\left|f(x)-f\left(x_{0}\right)\right| \geq \varepsilon\right\} \cap O_{\delta}\left(x_{0}\right)\right| \leq\left|E_{n}(\varepsilon) \cap O_{\delta}\left(x_{0}\right)\right|, \forall n \geq n_{\varepsilon} .
$$

Take $\forall n \geq n_{\varepsilon}$ and fix it. So, $f_{n_{0}} \in C_{s t}^{J}[a, b]$, then from (19) we obtain

$$
\lim _{\delta \rightarrow 0} \frac{\left|\left\{x:\left|f(x)-f\left(x_{0}\right)\right| \geq \varepsilon\right\} \cap O_{\delta}\left(x_{0}\right)\right|}{\left|O_{\delta}\left(x_{0}\right)\right|} \leq \lim _{\delta \rightarrow 0} \frac{\left|E_{n_{0}}(\varepsilon) \cap O_{\delta}\left(x_{0}\right)\right|}{O_{\delta}\left(x_{0}\right)}=0 .
$$

From the arbitrariness of $x_{0}$ it follows that $f \in C_{s t}^{J}[a, b]$. Theorem is proved.

Finally, compare the concept of $\mu$-stat continuity with the concept of approximate continuity. Let us recall the definition of approximate continuity.

Let $E \subset \mathbb{R}$ be some measurable (with respect to the Lebesgue measure) set and assume

$$
E\left(x_{0} ; h\right)=E \cap\left[x_{0}-h, x_{0}+h\right]=E\left[x_{0}-h, x_{0}+h\right] .
$$

Definition 7. The limit

$$
D_{x_{0}} E=\lim _{h \rightarrow 0} \frac{m E\left(x_{0} ; h\right)}{2 h},
$$

(in case it exists) is called a density of the set $E$ at the point $x_{0}$.

If $D_{x_{0}} E=1$, then $x_{0}$ is a point of density for the set $E$, and if $D_{x_{0}} E=0$, then $x_{0}$ is a rarefaction point of $E$.

In our case, $x_{0}$ is a point of $m$-stat density for the set $E$, where $m$ is a Lebesgue measure. The following theorem is known. 
Theorem 7. Almost all points of measurable set $E$ are its density points.

More details about the following concept can be found in [35].

Definition 8. Let the function $f(x)$ be given on the segment $[a, b]$ and $x_{0} \in[a, b]$. If there exists a measurable set $E \subset[a, b]$ with a density point $x_{0}$ such that $f(x)$ is continuous along $E$ at the point $x_{0}$, then $f(x)$ is said to be approximate continuous at the point $x_{0}$.

In our case, the concept of approximate continuity coincides with the one of m-stat continuity at the point $x_{0}$. Let us recall the following Denjoy theorem.

Theorem 8 (Denjoy). If $f(x)$ is a measurable and almost everywhere finite function in $[a, b]$, then it is approximate continuous at almost every point in $[a, b]$.

Consequently, if $f(\cdot)$ is measurable and almost everywhere finite in $[a, b]$, then it is $m$-stat continuous almost everywhere in $[a, b]$.

\section{Acknowledgements}

Author would like to express her deepest gratitude to Prof. B.T. Bilalov for his encouragement and valuable guidance throughout this research.

This work is supported by the Scientific and Technological Research Council of Turkey (TUBITAK) with Azerbaijan National Academy of Sciences (ANAS), Project Number 19042020 and by the Science Development Foundation under the President of the Republic of Azerbaijan, Grant No. EIF-BGM-4-RFTF1/2017-21/02/1-M-19.

The author thanks the anonymous referee for his careful reading the manuscript, for comments and suggestions.

\section{References}

[1] Altinok M., Kaya U., Kucukaslan M. $\alpha$-Statistical Supremum-Infimum and $\alpha$-Statistical Convergence. Azerb. J. Math. 2014, 4 (2), 31-42. doi:10.33773/jum.823084

[2] Balcerzak M., Dems K., Komisarski A. Statistical convergence and ideal convergence for sequences of functions. J. Math. Anal. Appl. 2007, 328, 715-729. doi:10.1016/j.jmaa.2006.05.040

[3] Basu A., Srivastava P.D. Statistical convergence on composite vector valued sequence space. J. Math. Appl. 2007, 29, 75-90.

[4] Bilalov B.T., Nazarova T.Y. Statistical convergence of functional sequences. Rocky Mountain J. Math. 2015, 45 (5), 1413-1423. doi:10.1216/RMJ-2015-45-5-1413

[5] Bilalov B.T., Nazarova T.Y. On Statistical Convergence in Metric Spaces. J. Math. Res. 2015,7 (1), $37-43$. doi:10.5539/jmr.v1n1p37

[6] Bilalov B.T., Nazarova T.Y. On the statistical type convergence and fundamentality in metric spaces. Caspian J. Appl. Math. Ecology and Economics 2014, 2 (1), 84-93.

[7] Bilalov B.T., Sadigova S.R. On $\mu$-statistical convergence. Proc. Amer. Math. Soc. 2015, 143, (9), $3869-3878$. doi:10.1090/S0002-9939-2015-12528-2

[8] Bruckner A.M. Differentiation of real functions. AMS, Providence, Rhode Island, USA, 1994. 
[9] Connor J.S. The statistical and strong p-Cesaro convergence of sequences. Analysis 1988, 8 (1-2), 47-63. doi: 10.1524/anly.1988.8.12.47

[10] Connor J.S. R-type summability methods, Cauchy criteria, P-sets and statistical convergence. Proc. Amer. Math. Soc. 1992, 115 (2), 319-327. doi:10.2307/2159248

[11] Connor J.S., Grosse-Erdmann K.G. Sequential definitions of continuity for real functions. Rocky Mountain J. Math. 2003, 33 (1), 93-121. doi:10.1216/rmjm/1181069988

[12] Duman O., Orhan C. $\mu$-statistically convergent function sequences. Czechoslovak Math. J. 2004, 54 (2), $413-422$.

[13] Eghbali N., Ganji M. Generalized Statistical Convergence in the Non-Archimedean L-fuzzy Normed Spaces. Azerb. J. Math. 2016, 6 (1), 15-22.

[14] Fast H. Sur la convergence statistique. Colloq. Math. 1951, 2, 241-244.

[15] Federer H. Geometric measure theory. Springer, New York, 1969.

[16] Fridy J.A. On statistical convergence. Analysis 1985, 5 (4), 301-313. doi:10.1524/anly.1985.5.4.301

[17] Fridy J.A. Statistical limit points. Proc. Amer. Math. Soc. 1993, 118 (4), 1187-1192. doi:10.2307/2160076

[18] Fridy J.A., Miller H.I. A matrix characterization of statistical convergence. Analysis 1991, 11 (1), 59-66. doi: 10.1524/anly.1991.11.1.59

[19] Fridy J.A., Khan M.K. Tauberian theorems via statistical convergence. J. Math. Anal. Appl. 1998, 228 (1), $73-95$. doi:10.1006/jmaa.1998.6118

[20] Fridy J.A., Orhan C. Lacunary statistical summability. J. Math. Anal. Appl. 1993, 173 (2), 497-504. doi: 10.1006/jmaa.1993.1082

[21] Fridy J.A., Orhan C. Lacunary statistical convergence. Pacific J. Math. 1993, 160 (1), 43-51.

[22] Gadjiev A.D., Orhan C. Some approximation theorems via statistical convergence. Rocky Mountain J. Math. 2002, 32 (1), 129-138. doi:10.1216/rmjm/1030539612

[23] Gadjiev A.D., Ghorbanalizadeh A.M. On the A-statistical approximation by sequences of k-positive linear operators. Proc. Inst. Math. Mech. Natl. Acad. Sci. Azerb. 2009 31, 41-52.

[24] Gadjiev A.D. Simultaneous statistical approximation of analytic functions and their derivatives by $k$-positive linear operators. Azerb. J. Math. 2011, 1 (1), 57-66.

[25] Gordon R. The integrals of Lebesgue, Denjoy, Perron and Henstock. In: Graduate studies in mathematics, 4. American Mathematical Society, 1994.

[26] Hazarika B., Savaş E. $(\lambda ; \mu)$-statistical convergence of double sequences in n-normed spaces. Note di Mathematica, 2012, 32 (2), 101-114.

[27] Jasinki J., Reclaw I. Ideal convergence of continuous functions. Topology Appl. 2006, 153 (18), $3511-3518$. doi:10.1016/j.topol.2006.03.007

[28] Komisarski A. Pointwise $\mathscr{I}$-convergence and $\mathscr{I}$-convergence in measure of sequences of functions. J. Math. Anal. Appl., 2008, 340 (2), 770-779. doi:10.1016/j.jmaa.2007.09.016

[29] Lahiri B.K., Chakrabarti S. Approximately continuous functions in a measure space. Vietnam J. Math. 1997, 25 (1), $59-64$.

[30] Mačaj M., Šalát T. Statistical convergence of subsequences of a given sequence. Math. Bohem. 2001, 126 (1), $191-208$. doi:10.21136/MB.2001.133923

[31] Maddox I.J. Statistical convergence in a locally convex space. Math. Proc. Cambridge Philos. Soc. 1988, 104 (1), 141-145. doi:10.1017/S0305004100065312

[32] Móricz F. Statistical limits of measurable functions. Analysis 2004, 24, 1-18. doi:10.1524/anly.2004.24.1.1 
[33] Mursaleen M. A-statistical convergence. Math. Slovaca 2000, 50 (1), 111-115.

[34] Mursaleen M., Mohiuddine S.A. Statistical convergence of double sequence in intuitionistic fuzzy normed spaces. Chaos Solitons Fractals 2009, 41, 2414-2421. doi:10.1016/j.chaos.2008.09.018

[35] Natanson I.P. Theory of Functions of a Real Variable, Nauka, Moscow, 1974. (in Russian)

[36] Patterson R.F., Savaş E. Lacunary statistical convergence of double sequences. Math. Commun. 2005, 10, 55-61.

[37] Rath D., Tripathy B.C. On statistically convergent and statistically Cauchy sequences. Indian J. Pure Appl. Math. 1994, 25 (4), 381-386.

[38] Šalát T. On statistically convergent sequences of real numbers. Math. Slovaca 1980, 30 (2), 139-150.

[39] Sarabadan S., Talebi S. Statistical convergence and ideal convergence of sequences of functions in 2-normed spaces. Int. J. Math. Math. Sci. 2011, 2011, 1-10. doi:10.1155/2011/517841

[40] Savaş E. On lacunary strong $\sigma$-convergence. Indian J. Pure Appl. Math. 1990, 21 (4), 359-365.

[41] Savaş E., Borgohain S. On strongly almost lacunary statistical A-convergence defined by Musielak-Orlicz function. Filomat 2016, 30 (3), 689-697. doi:10.2298/FIL1603689S

[42] Schoenberg I.J. The integrability of certain functions and related summability methods. Amer. Math. Monthly 1959, 66, 361-375. doi:10.1080/00029890.1959.11989303

[43] Steinhaus H. Sur la convergence ordinaire et la convergence asumptotique. Colloq. Math. 1951, 2, 73-74.

[44] Zygmund A. Trigonometric series, Vol. II. Cambridge Univ. Press, London-New York, 1979.

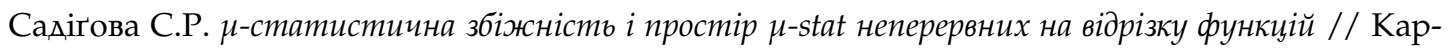
патські матем. публ. - 2021. - Т.13, №2. - С. 433-451.

У цій статті введено поняття точкової $\mu$-статистичної щільності, на основі чого визначено поняття точкової $\mu$-статистичної границі, що генерується деякою мірою Бореля $\mu(\cdot)$. Також ми вводимо поняття $\mu$-статистичної фундаментальності в точці та доводимо ії еквівалентність з $\mu$-stat збіжністю. Класифікація точок розриву перенесена на цей випадок. Визначено відповідний простір $\mu$-stat неперервних на відрізку функцій з sup-нормою. Аоведено, шо цей простір є банаховим та розглянуто зв'язок між цим простором та простором неперервних і сумовних за Аебегом функцій.

Ключові слова і фрази: $\mu$-stat збіжність, $\mu$-stat фундаментальність, простір $\mu$-статистично неперервних функцій. 